\title{
Community-acquired Klebsiella pneumoniae systemic infection complicated with rhombencephalitis
}

\author{
Delia Stanca ${ }^{1}$, Daniela Bala ${ }^{2}$, Mihaela Rusca ${ }^{2}$, Andra Achim² ${ }^{2}$, and Dafin Muresanu ${ }^{1}$ \\ ${ }^{1}$ UMF Iuliu Haţieganu Cluj-Napoca \\ ${ }^{2}$ Cluj-Napoca County Emergency Hospital
}

July 2, 2020

\begin{abstract}
Rhombencephalitis refers to inflammatory diseases of the rhombencephalon. The encephalitic processes located to the rhombencephalon are frequently associated with Listeria monocytogenes and enterovirus infections. We report a case of communityacquired Klebsiella Pneumoniae systemic infection with CNS involvement in an adult patient without significant comorbidities.
\end{abstract}

Community-acquired Klebsiella pneumoniae systemic infection complicated with rhombencephalitis Delia-Maria Stanca ${ }^{\mathrm{a}, \mathrm{b}}$, Daniela Larisa Bala ${ }^{\mathrm{b}}$ (corresponding author*), Mihaela Ruscã ${ }^{\mathrm{b}}$, Andra Achim ${ }^{\mathrm{b}}$ Dafin Fior Mureșanu ${ }^{\mathrm{a}, \mathrm{b}}$

${ }^{\text {a }}$ Iuliu Hațieganu of and Pharmacy , 43, 400012

b Cluj-Napoca County Emergency Hospital, Neurology Department, Victor Babeș Street No. 43, 400012 Cluj-Napoca, Romania

${ }^{*}$ Corresponding author details:

-mailing address: , Neurology Department, Victor Babeș Street No. 43400012

-email address:bala.daniella@yahoo.com

Key clinical message

CNS involvement in community-acquired Klebsiella pneumoniae infection is rare and usually presents as abscess or meningitis, while, to our knowledge, no cases of K. Pneumoniae infection complicated with rhombencephalitis have been previously reported.

Abstract

Rhombencephalitis refers to inflammatory diseases of the rhombencephalon. The encephalitic processes located to the rhombencephalon are frequently associated with Listeria monocytogenes and enterovirus infections. We report a case of community-acquired Klebsiella Pneumoniae systemic infection with CNS involvement in an adult patient without significant comorbidities.

Introduction

The term rhombencephalitis (RE) refers to inflammatory diseases involving the hindbrain (brainstem and cerebellum).

It was described for the first time by two scientists, Edwin Bickerstaff and Philip Cloake in $1951^{(1,2)}$ to designate inflammatory affections of the rhombencephalon. 
Rhombencephalitis has a wide range of etiologies, which can be divided into infectious, autoimmune and paraneoplastic causes.

The most common infectious agent is Listeria monocytogenes, followed by Enterovirus 71 and herpes viruses. The most common autoimmune cause is Behcet's disease and the most frequent paraneoplastic syndrome is caused by small cell lung cancer. ${ }^{(3)}$

In Gram-negative CNS infections, a primary focus may be found in neonates, traumatic or neurosurgical patients, but in adults without a history of surgical operations the primary focus of the infection could not be detected in up to $60 \%$ of cases. ${ }^{(4)}$

The most common presentations of Klebsiella pneumoniae (K.pneumoniae) CNS infection in adult are meningitis and brain abscess, but cerebritis and cerebral hemorrhage were also reported. (5)

Here, we report a case of K. pneumoniae septicemia associated with CNS involvement in an immunocompentent patient, without neurosurgical or trauma history.

Case presentation

We present a case of an immunocompetent 60 years old male patient admitted to the Neurology Department with sudden onset of difficulty in walking, poor balance, slurred speech and complaints of mild headache.

His past medical history included hypertension and a chronic right sciatica, for which he received symptomatic treatment with NSAIDs and paravertebral infiltration with steroids.

At the time of admission, he was apyrexial, alert and with no signs of meningism. No facial asymmetry or palpeberal ptosis was noted. He had bilateral gaze-evoked nystagmus, gait ataxia, bilateral cerebellar dysmetria and dysarthria. Laboratory findings: minimal hepatic cytolysis, inflammatory syndrome with leucocytosis $\left(\mathrm{WBC}=22 \times 10^{9}\right.$ cells $/ \mathrm{L}, 90 \%$ neutrophils, erythrocyte sedimentation rate $=40 \mathrm{~mm} / \mathrm{h}$ and Creactive protein $=18 \mathrm{mg} / \mathrm{L})$.

An emergency brain CT angiography was performed and no acute lesions were observed.

At first, an ischemic stroke in the vertebrobasilar region was suspected and treatment with antiplatelet and statins was initiated.

Within a few hours of hospitalization, his condition worsened, he presented several episodes of projectile vomiting and became less responsive (somnolence alternating with agitation). Further on, his neurological status deteriorated rapidly and he developed tetraplegia. He became comatose (GCS=3 points), with nonreactive mydriatic pupils and was immediately transferred to the intensive care unit (ICU).

Screening for MRSA, ESBL, VRE, and CRE was performed upon ICU admission, with negative results.

Blood cultures were also taken within the first 24 hours of admission.

In the ICU department the patient became febrile, with marked inflammatory syndrome. An encephalitic process was suspected and empiric antibiotic therapy was initiated with Vancomycin, Ampicillin and Meropenem, associated with Acyclovir.

He tested negative for HIV and syphilis.

Cerebrospinal fluid examination revealed a sanguinolent aspect of the CSF, mild elevated leukocyte counts, elevated protein levels and normal CSF/serum glucose ratio.

The patient was referred for an emergent brain MRI which showed bilateral cerebellar lesions, hyperintense in T2/FLAIR, diffuse edema involving bilateral cerebellum, medulla and pons, aspect suggestive for encephalitis. Neither contrast enhanced lesion, nor leptomeningeal enhancement were shown.

Chest radiograph was normal. 
Cultures of CSF were positive for K. pneumoniae spp and it was detected in blood and urine cultures as well, on the 5th day of admission. The isolated strain was susceptible to the majority of antibiotics.

Therefore, a diagnosis of systemic infection with K. pneumoniae complicated with rhombencephalitis was made.

The antibiotic treatment was adjusted according to the results of blood, urine and CSF cultures.

A abdomino-pelvic CT scan was performed, to rule out neoplastic disease, which could cause immune suppression. The examination revealed right iliopsoas muscle abscess. An ultrasound guided drainage was performed and the pus culture was also positive for K. pneumonie.

A second screening for multi-drug resistance bacteria was performed 9 days after ICU admission and Klebsiella CRE and ESBL was detected on perianal and rectal swabs.

Outcome and follow-up

Despite intensive treatment, the patient's condition did not improve and he died on the 14th day of hospitalization.

Discussion

Our patient was a 60-year-old male with no previous hospital admissions and no obvious risk factors for suppressed immune status, who developed a systemic infection with CNS involvement, with an unusual pathogen.

Rhombencephalitis is usually caused by Listeria monocytogenes, followed by herpes simplex virus (HSV) and Enterovirus-71, while to our knowledge, no cases of K. Pneumoniae infection with CNS involvement presenting as rhombencephalitis have been previously reported.

The differential diagnosis of rhombencephalitis should include multiple sclerosis, Behcet's disease, paraneoplastic syndrome and other infectious diseases (Listeria monocytogenes, EBV, Mycoplasma etc). ${ }^{(6)}$

Listerial rhombencephalitis has a characteristic biphasic course: for the first 2-4 days there are nonspecific symptoms, such as fever, headache, nausea and vomiting, followed by progressive asymmetrical cranial nerve palsies, cerebellar signs, hemiparesis and impairment of consciousness. The onset of neurological deterioration is usually abrupt. The syndrome progresses rapidly and sometimes has a fatal outcome. (7)

In our case, the course of the syndrome was different, with mild headache presenting as the first symptom, followed by cerebellar signs, impairment of consciousness, quadriplegia and ultimately fever, with rapid progression.

The diagnosis is usually achieved by MRI findings and confirmed by CSF analysis in correlation with clinical manifestations.

Radiological findings reveal high signal intensities on T2-weighted, FLAIR and DWI (hypointese on ADC map) images in the brainstem and cerebellum, with hypo- or isointense signal in T1. On Postgadolinium T1-weighted series, there is variable enhancement, depending on the etiology. MRI findings are not exclusive of any particular entity. ${ }^{(8)}$

CSF examination usually reveals moderate pleocytosis, normal/reduced glucose concentration, elevated proteins. Positive CSF and blood cultures are the most specific findings for diagnosis.

In this case, CT scan upon admission was unremarkable, which is possible in the early course of the disease. When the clinical status of the patient deteriorated, an MRI was performed, which evidenced lesions characteristic for rhombencephalitis.

Community-acquired bacteremia is defined as a positive blood culture taken on or within 48 hours of admission. ${ }^{(9)}$ In our case, the blood cultures were taken within 24 hours of admission. 
Combining CSF, blood and urine cultures, with radiological and clinical findings, we established the diagnosis of community-acquired K. pneumoniae septicemia complicated with rhombencephalitis.

Klebsiella species are Gram-negative bacteria, found ubiquitously in nature, including in plants, animals, and humans. It is the causative agent of several types of infections in humans, including respiratory tract infections, urinary tract infections (UTIs) and bloodstream infections (BSI). ${ }^{(10)}$

K.pneumoniae strains can be divided into opportunistic, hypervirulent and multidrug-resistant groups. Most frequently, opportunistic strains cause hospital-acquired infection in patients with impaired host defenses. (11)

Hypervirulent strains of $\mathrm{K}$. Pneumoniae (Kp) affect healthy people in community settings and can cause severe infections, such as pyogenic liver abscess, endophthalmitis and meningitis. ${ }^{(12)}$

In recent years, new hypervirulent Kp strains have been associated with life-threatening infections with distant metastasis and, despite the presence of comorbidities in most patients, it is seen in younger, healthy patients as well. $(13,14)$

Diabetes has been speculated as a significant risk factor of hvKP infection. ${ }^{(15,16)}$ It has been shown that infective and proinflammatory properties of Klebisella are more virulent in the alcoholic host. (17) Our patient had no history of diabetes or alcohol abuse.

The frequency of Klebsiella as a CNS pathogen is increasing, with no improvement in prognosis over a 15-year period despite the availability of newer antibiotics. Particularly high mortality with Klebsiella septicemia and CNS spread has been noted. ${ }^{(18)}$

K. pneumoniae is the second leading cause of (BSI) caused by Gram-negative bacteria. $(10,19)$

BSI can be primary (no identifiable source) or, more often, secondary infection through dissemination from a known source. The most common sources of secondary BSI include the urinary tract, the gastrointestinal tract, intravenous or urinary catheters and respiratory sites. ${ }^{(20)}$

K. pneumoniae psoas muscle abscess is a rare infection that has been described in case reports and small institutional series, mostly from Taiwan, where, since 1981, a distinctive syndrome of community-acquired K. pneumoniae septicemia with liver abscess has been reported. ${ }^{(21,22)}$ Among those cases, 43 percent had concurrent urinary infection. This syndrome is notable for high mortality (10 to 40\%), and some cases have been complicated by meningitis or endophthalmitis. ${ }^{(23)}$

The psoas abscess is considered primary if the cause is hematogenous seeding from a distant site by bacteremia or sepsis, and secondary if there is a contiguous infectious source from vertebrae, pancreas, kidney, ureter, appendix, bowel, or hip joint. ${ }^{(24)}$

The portal of entry of K. pneumoniae bacteremia is identified in only $32 \%$ of cases; it is usually urinary, digestive and hepato-biliary. Other gateways - vascular, oral or lung, were rarely found. ${ }^{(25)}$

In our case, it was difficult to establish the primary source of the systemic infection.

The psoas major originates along the outer surfaces of the vertebral bodies of T12 and L1-L3 and their associated intervertebral discs.

Taking into consideration the anatomy of the psoas muscle and the fact that the patient received paravertebral infiltration in the lumbar region for his sciatica, we can not rule out the hypothesis that the psoas abscess was the primary source of infection.

Intramuscular injections can rarely result in serious infectious complications such as abscesses which may progress to bacteriemia and generalized sepsis.

Due to the proximity of iliopsoas abscess to the vertebrae, it is possible that the adjacent structures could be infected via direct invasion, causing epidural abscess, osteomyelitis or discitis. In this case, the abdomino- 
pelvic CT scan did not evidence any of this. We can not exclude that the lumbar sciatic was caused in the first place by an infectious process in the lumbar region.

Another hypothesis is that the primary source of infection could be the urinary tract infection, which could led to psoas abscess either by contiguity or hematogenous spreading, with CNS involvement.

Management of rhombencephalitis includes treating the underlying cause in addition to supportive management.

Carbapenems are typically the drug of choice to treat severe infections caused by ESBL-producing bacteria.

Due to the selective pressure of treating ESBL infections with carbapenems, resistance has emerged and K. pneumoniae is the most common carbapenem-resistant Enterobacteriaceae (CRE).

Because Listeria and HSV are the most common treatable acute causes, it is recommended to start empiric therapy with ampicillin and acyclovir for all cases after CSF and blood samples have been obtained for cultures and the polymerase chain reaction (PCR). Antibiotics can be changed based upon MRI, culture results, PCR results. ${ }^{(26)}$

ICU-acquired infection is defined if the pathogen colonies are detected on screening swabs samples 7 days after ICU admission, in previously negative patients. ${ }^{(27)}$ In our case, community-acquired bacteriemia was associated with an ICU-acquired infection (K.pneumoniae CRE and ESBL), leading to poor prognosis.

Given the aggressive course of the disease and the lack of response to antibiotic treatment, we presume that a hypervirulent strain of $\mathrm{K}$. pneumoniae was involved in this case.

CNS infections represent a challenge for clinicians because of the poor concentration achieved by antibiotics in the CSF. In particular, if the isolated microorganism exhibits multiple resistances, the situation becomes critical because of the relative difficulty of reaching the high concentrations needed.

Despite advances in microbiology and imaging, rhombencephalitis can be a diagnostic challenge due to the multitude of possible differential diagnoses and the difficulty to determine etiology. Rapid diagnosis and adequate treatment are important to reduce mortality and morbidity. ${ }^{(28)}$ Overall mortality in rhombencephalitis is around $10-15 \%$. (29)

A particularity of this case was the systemic infection with K.pneumoniae and CNS involvement presenting as rhombencepahalitis in an immunocompetent patient, without known comorbidities or risk factors that could affect immunocompetence.

Another particularity of the case was the difficulty to establish the primary source of K.pneumoniae systemic infection, which remained unknown.

Conclusion

This case shows an unusual and severe CNS complication of a systemic infection with K. pneumoniae rhombencephalitis, which, to our knowledge, has not been reported before.

It illustrates the difficulty of identifying the original site of infection in the presence of several infected sites and bacteriemia.

It also shows the devastating course that community-acquired K. Pneumoniae infection can have in an apparently immunocompetent patient, without significant comorbidities.

Author contribution

Delia Maria Stanca: Consultant neurologist in charge of the patient. Given approval of the final version of the paper.

Daniela Larisa Bala: Resident doctor, conception and design of the case report.

Mihaela Ruscă: Resident doctor, drafting the work and revising it for important intellectual content. 
Andra Achim: Resident doctor, comparing the case report to the current literature.

Dafin Fior Mureșanu: Coordinator of the team.

Acknowledgments

Many thanks to Ioana Robu, Iuliu Hatieganu University of Medicine and Farmacy of Cluj, Romania, for the help in editing this manuscript.

References

1. Moragas M, Martinez-Yelamos S, Majos, Fernandez-Viladrich P, Rubio F, Arbizu T. 2011. Rhombencephalitis, A Series of 97 Patients. Medicine 90: 256Y261.

2. Pakize Ö. Yýlmaz, Nevzat M et al. 2016. Linezolid and dexamethasone experience in a serious case of listeria rhombencephalitis. J Infect and Public Health 9: 670-674.

3. Diallo IM, Boubacar S, Mansaré, ML et al. 2017. Diagnosis approach and management of rhombencephalitis: literature review. Clinical Neurology and Neuroscience 1(2):47-51. doi: 10.11648/j.cnn.20170102.15

4. Berk SL, McCabe WR. 1980. Meningitis caused by gram-negative bacilli. Ann Intern Med 93(2):253-60.

5. Lin CH, Lu CH, Lui CC, Huang CR, Chuang YC, Chang WN. 2010. Protean neuroimaging presentations in an adult with Klebsiella pneumoniae infection. Acta Neurol Taiwan 19(3): 199-203.

6. Christo PP, Silva JS, Werneck IV, Dias SL. 2010. Rhombencephalitis possibly caused by Mycoplasma pneumoniae. Arq Neuropsiquiatr 68(4):656-8.

7. R.W. Armstrong, P.C. Fung. 1993. Brainstem encephalitis (rhombencephalitis) due to Listeria monocytogenes: case report and review. Clin Infect Dis 16:689-702.

8. Sharma R, Di Muzio B et al. Rhombencephalitis. Radiopaedia [Internet]. Available from: https://radiopaedia.org/articles/rhombencephalitis

9. Ko WC, Paterson DL, Sagnimeni AJ et al. 2002. Community-acquired Klebsiella pneumoniae bacteremia: global differences in clinical patterns. Emerg Infect Dis. 8(2):160-166. doi:10.3201/eid0802.010025.

10. Podschun R, Ullmann U. 1998. Klebsiella spp. as nosocomial pathogens: epidemiology, taxonomy, typing methods, and pathogenicity factors. Clin Microbiol Rev 11(4):589-603.

11. Calfee DP. 2017. Recent advances in the understanding and management of Klebsiella pneumoniae. F1000Res 6:1760. doi: 10.12688/f1000research.11532.1

12. Martin RM, Bachman MA. 2018. Colonization, infection, and the accessory genome of Klebsiella pneumoniae. Front Cell Infect Microbiol 8:4. doi:10.3389/fcimb.2018.00004).

13. Prokesch BC, Tekippe M, Kim J et al. 2016. Primary osteomyelitis caused by hypervirulent Klebsiella pneumoniae Lancet Infectious Diseases 16(9): e190-e195.

14. Fang CT, Chuang YP, Shun CT, Chang SC, Wang JT. 2004. A novel virulence gene in Klebsiella pneumoniae strains causing primary liver abscess and septic metastatic complications. J of Exp Medicine 199(5):697-705, 2004.

15. Ko WC, Paterson DL, Sagnimeni AJ et al. 2002. Community-acquired Klebsiella pneumoniae bacteremia: global differences in clinical patterns. Emerg Infect Dis 8:160-6.

16. Cheng DL, Liu YC, Yen MY, Liu CY, Wang RS. 1991. Septic metastatic lesions of pyogenic liver abscess. Their association with Klebsiella pneumoniae bacteremia in diabetic patients. Arch Intern Med 151:1557-9.

17. Chang WN, Lu CH, Chang CS, Huang CR. 2003. Community-acquired spontaneous bacterial meningitis in patients with alcoholic liver disease. J Formos Med Assoc 102(9):653-5. 
18. Lu CH, Chang WN, Chuang YC, Chang HW. 1998. The prognostic factors of adult gram-negative bacillary meningitis. J Hosp Infect 40(1):27-34.

19. Magill SS, Edwards JR, Bamberg W et al. 2014. Emerging Infections Program Healthcare-Associated Infections and Antimicrobial Use Prevalence Survey Team.N Engl J Med 370(13):1198-208.

20. Montgomerie JZ, Ota JK. 1980. Klebsiella bacteremia. Arch Intern Med 140(4):525-7.

21. Jang TN, Juang GD, Fung CP. 1997. Fulminating gas-forming psoas muscle abscess due to Klebsiella pneumoniae following a deep neck infection. J Formos Med Assoc 96:134.

22. Cheng, D. L., Y. C. Liu, M. Y. Yen, C. Y. Liu, and R. S. Wang. 1991. Septic metastatic lesions of pyogenic liver abscess. Their association withKlebsiella pneumoniae bacteremia in diabetic patients. Arch. Intern. Med 151: 1557-1559.

23. Liu YC, Cheng DL, Lin CL. 1986. Klebsiella pneumoniae liver abscess associated with septic endophthalmitis. Arch Intern Med 146:1913-1916.

24. Taiwo B. 2001. Psoas abscess: a primer for the internist. South Med J 94(1):2-5, 2001.

25. Fang CT, Chang SC, Hsueh PR, Chen YC, Sau WY, Luh KT. 2000. Micrpbiological features of community-acquired bacterial meningitis: study at a university hospital in Taiwan. J Formos Med Assoc 99(4):300-304

26. Jubelt B, Mihai C, Terrence L, Padma V. (2011). Rhombencephalitis / brainstem Encephalitis. Current neurology and neuroscience reports. 11. 543-52. 10.1007/s11910-011-0228-5.

27. Zaha DC, Kiss R, Hegedus C, et al. 2019. recent advances in investigation, prevention, and management of healthcare-associated infections (HAIs): resistant multidrug strain colonization and its risk factors in an Intensive Care Unit of a University Hospital. Biomed Res Int 9:2510875. doi:10.1155/2019/2510875.

28. Mansbridge CT, Grecu I, Li Voon Chong JS, Vandervelde C, Saeed K. 2017. Two cases of listeria rhombencephalitis. IDCases. 11:22-25. doi:10.1016/j.idcr.2017.12.002

29. Duarte J, Rossatto L, Fernandes J et al. 2014. Rhombencephalitis: a pictorial review.. ECR Educational Exhibit /C-1367.

Figure 1: MRI axial fluid-attenuated inversion recovery (FLAIR) sequence (A, B) and diffusion weighted image (DWI) sequence (C, D) showing bilateral, asymmetric, hyperintensity in the cerebellum with extension into the brainstem $(\mathrm{A}, \mathrm{C})$. 


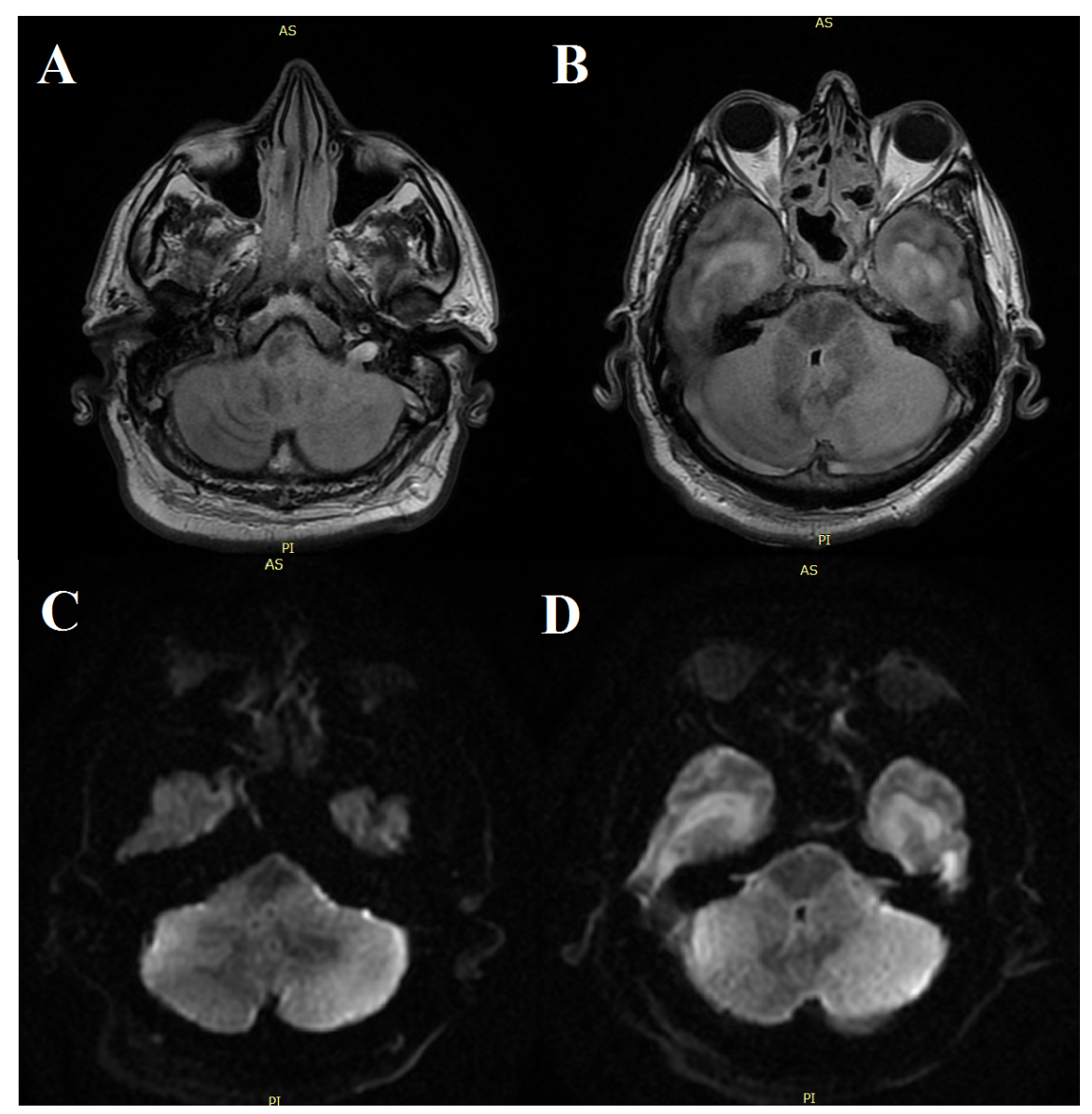

